\title{
(NARRAR) FICCIONALIZAR LAS NORMAS ESCOLARES. UNA EXPERIENCIA DE FORMACIÓN DE SUBJETIVIDADES POLÍTICAS CON ESTUDIANTES DE BACHILLERATO'
}

\section{(NARRATE) FICTIONALIZE SCHOOL NORMS. AN EXPERIENCE OF POLITICAL SUBJECTIVITIES FORMATION WITH HIGH SCHOOL STUDENTS}

\section{Edisson Leonardo Parra Herrera**}

Universidad de Los Andes.

"la apertura hacia otras cosmologías, hacia otras sensibilidades y formas de vida, es una condición básica del respeto activo, del debido reconocimiento, de la auténtica alteridad. La emergencia de otros relatos, su puesta en diálogo, nos permite, también, apreciar lo nuevo como lo otro que nos constituye, esto es, como posibilidad de ser".

(Ruiz y Prada, 2012, p. 25)

\section{RESUMEN}

Este documento presenta los resultados de una investigación educativa enfocada a la formación política mediante el uso de narraciones (auto) biográficas. Así, muestra la aplicación de la estrategia didáctica "me en-video con las normas escolares, me en-video con la escuela" a partir de la que se construyeron narrativas escritas y audiovisuales sobre las experiencias con las normas escolares. Pedagógicamente el 
trabajo estuvo dirigido a formar una consciencia de poder o pensamiento político que permitiera al grupo transfigurar las formas de verse y de relacionarse. Investigativamente se enfocó en determinar los límites y potencialidades de las narraciones en el aula para formar la subjetividad política. En concreto, dirigió la mirada a la ficción, como componente particular de la narración, para explorar su potencia en la descripción, reinterpretación y proyección de nuevas formas de ser y de estar. De esta manera, se responde a la necesidad de dar lugar a otros tipos de pensamiento, distantes de la racionalidad lógica instrumental, para comprender el mundo de la escuela. Se concluye, principalmente, que las herramientas (auto)biográfico-narrativas no garantizan por sí mismas la formación de elementos que configuren subjetividades políticas. Para ello, se requiere, además de espacios de movilización social, que estén articuladas a unos objetivos formativos, pedagógicos y políticamente delimitados. Mediante la producción y circulación de los relatos, gracias al elemento ficcional, se construyen las bases para transfigurar las identidades colectivas, las maneras de verse a sí mismos y de relacionarse con los demás, así como la construcción de un modo de pensar político.

PALABRAS CLAVE: Investigación (auto) biográfico-narrativa, formación política, subjetividad política, voluntad de ficción, normas escolares, alteridad.

\section{ABSTRAC}

This document presents the results of an educational research focused on the political formation though the use of (auto) biographic narratives. Thus, it shows the application of the teaching and learning strategy, "me en-video con las normas escolares, me en-video con la escuela", from which written and audiovisual narratives are built up about the experiences with school norms. Pedagogically, the research aimed at creating awareness of the political power or thought that allows the group to transfigure the way they see and relate with each other. For research purposes, the focus laid on determining the potentialities and limitations of the classroom narratives to form political subjectivity. Specifically, it draws attention to the fiction as a particular component of the narratives to explore its power in the description, reinterpretation and projection of new ways of being. By doing so, it responds to the need of generating other types of thought beyond instrumental rationality to understand the school world. It is concluded that the (auto) biographical-narrative tools do not guarantee by themselves the formation of elements that configure political subjectivities. For this, it is additionally required to have spaces for social mobilization articulated to formative, pedagogical and politically delimited objectives. Through the production and circulation of stories, thanks to the fictional element, the bases are built to transfigure collective identities, the ways of seeing themselves and relating to others, as well as the construction of a political way of thinking.

KEYWORDS: (Auto) biographic narratives research; Political Formation; Political subjectivity; Will to Fiction; School norms; Alterity.

\section{INTRODUCCIÓN}

Las metodologías narrativas, (auto)biográficas y biográficas adquieren cada vez más fuerza en el campo educativo como corriente de investigación, formativa y pedagógica (Gil, 1997; Murillo, 2016). Este enfoque de investigación, que se adscribe a los métodos cualitativos y hermenéuticos, tiene sus antecedentes en los estudios adelantados por la Escuela de Chicago en 1930. De igual forma, está fundamentado filosóficamente en el giro hermenéutico y el giro lingüístico. Así, Bolívar, Domínguez y Fernández (2001) muestran cómo en los sesenta se produce, en las ciencias sociales, un giro desde la perspectiva positivista a una 'perspectiva 
interpretativa' en la que 'el significado de los agentes se convierte en el foco central de la investigación" (p. 52). Por tanto, en la indagación narrativa en educación las múltiples disciplinas sociales y humanas "se interesan en los modos como los humanos dan significado a su mundo mediante el lenguaje" (p. 53).

Así, en el campo educativo, la investigación biográfico-narrativa se interesa por rescatar la vida y las voces de los y las docentes. De este modo, se intenta construir conocimiento desde la experiencia al tiempo que se interpela a la reflexión a los actores educativos sobre su vida y prácticas escolares. En este proceso, el lenguaje propio cumple un papel fundamental que permite no solamente comprender los procesos y vidas educativas, sino que también es el elemento que sirve para su transfiguración. De esta manera, "las narrativas propias, cuando no se quedan solo en un recuento enunciativo de experiencias pasadas, pueden tener una función performativa" (Bolívar, et al., 2001, p. 66). Sin embargo, hay que señalar que las narrativas se presentan como ambivalentes. Pues, si bien los relatos (auto)biográficos son formas de contracultura y herramientas que potencian una nueva 'política de la identidad', del mismo modo son formas de gobernabilidad de los individuos. Dado que, "son también un dispositivo de saber y de poder, y como tales a la vez son instrumentos de dominio mediante el acceso al conocimiento a su vida" (Huchim y Reyes, 2013, p. 16). Por ello, si se ignora que pueden hacer parte de "las fuerzas políticas y sociales que condicionan la individualidad, sirve de coartada para mantener el statu quo" (Bolívar, et al., 2001, p. 68).

En este sentido, los relatos forman la subjetividad. Esta configuración del sujeto por medio de la narración se vincula con la configuración de la trama. Es decir, cuando contamos historias nos estamos constituyendo a nosotros mismos.
La narración es la posibilidad que tenemos de contar historias mediante las cuales les damos a nuestras vidas una orientación en el tiempo. La narración, entonces nos permite comprendernos y hacer-nos sujetos históricos, a la vez que nos abre la idea de proyecto, de ir más allá de las circunstancias del presente y de los aconteceres de la vida cotidiana. (Ruiz y Prada, 2012, p. 50)

Es así como, en los espacios formativos los relatos permiten desarrollar en los sujetos elementos que podemos considerar constitutivos de la subjetividad política como la memoria, el olvido, el posicionamiento, la consciencia, la narración, la identidad y la proyección (Ruiz y Prada, 2012). Esto implica, como consecuencia, que al reconocerse como parte de los entramados de poder, por medio de las narrativas, puede formarse un pensamiento político o consciencia de poder.

En todo caso, no es suficiente con la introducción de las herramientas narrativas en el aula para formar determinados elementos en los sujetos|. Así, es necesario que estas se encuentren articuladas a un proyecto con objetivos pedagógicos o formativos claramente delimitados. Precisamente, en la investigación reportada, se buscó la formación de subjetividades políticas a través de una estrategia didáctica que tuvo como finalidad formar un pensamiento político que implicara formas otras de mirarse, de relacionarse consigo mismo y con los otros.

Por tanto, el objetivo de la investigación fue comprender las potencialidades y limitaciones de las narrativas (auto)biográficas para formar en un grupo de estudiantes una consciencia de poder o pensamiento político. Esto es, la posibilidad de interpretarse como parte de los entramados de poder, como un ser que no puede ser, sino con otros y que se entiende en constante transformación. El proyecto fue 
implementado en un colegio distrital de la ciudad de Bogotá desde el año 2017 con el grado octavo hasta el año 2020 cuando terminaron el bachillerato.

\section{ANTECEDENTES DE LA FORMACIÓN DE LA SUBJETIVIDAD POLÍTICA A PARTIR DE LAS METODOLOGÍAS (AUTO)BIOGRÁFICO NARRATIVAS: FICCIÓN Y SENTIDO}

Los estudios narrativos y biográficos abordan un campo de acción y de reflexión amplio y variado. Así, se agrupan en ellos disciplinas que van desde las ciencias sociales a la enfermería (Velasco, 2019). En todo caso, existe como punto nodal la mirada hacia la experiencia y la vida de los sujetos. Según Huchim y Reyes (2013), el método narrativo se plantea desde "un enfoque en la experiencia, el tiempo, el conocimiento personal, y la reflexión y deliberación" (p. 9). En consecuencia, en la esfera educativa, los procesos formativos son remitidos a la relación entre el aprendizaje y las biografías particulares. Pues, "todo trayecto de vida es un trayecto de formación, en el sentido en que organiza temporal y estructuralmente las adquisiciones y los sucesivos aprendizajes en el ámbito de una historia" (Delory-Momberger, 2009, p. 25). De este modo, las ciencias de la educación abordan los 'trayectos de formación', pero no buscan

tanto producir un saber objetivado en una u otra de esas disciplinas como si comprender, a través de la manera por la cual los actores de la escuela muestran sus experiencias de formación y aprendizaje, el papel representado por la escuela $y$ las instituciones educativas en las construcciones biográficas individuales $y$ en los procesos de socialización. (DeloryMomberger, 2009, p. 25)

Por tanto, en la escuela convergen conocimientos especializados y saberes de experiencia que inciden en la configuración de los sujetos. Precisamente, las narrativas son reconocidas como dispositivos de formación de subjetividades. En consecuencia, se encuentran trabajos de formación e investigación que desde las metodologías biográfico-narrativas apuntan a la configuración de aspectos que se consideran constitutivos de la subjetividad política como la identidad, la memoria, la producción de sentido o la posibilidad del uso de la ficción.

De este modo, en la esfera educativa se trabaja como uno de los elementos más sobresalientes de las narrativas el potencial que tienen los relatos (auto) biográficos para configurar identidades (Gil, 1997). Dicha construcción de identidad no puede verse como neutral y por tanto debe entenderse "mediante el reconocimiento de las luchas por el poder $\mathrm{y}$ de las situaciones alienantes desde las que (...) cualquier escolar ha de enfrentarse para llegar a ser él mismo" (Gil, 1997, p. 127). En este orden de ideas, los relatos se constituyen en herramientas que posibilitan a los sujetos reconocerse como parte de los entramados de poder y producir que tomen una posición frente a los diferentes acontecimientos. Es así como, las narraciones permiten reconocer lenguajes alternativos al institucional, tanto como las marcas que dejan los lenguajes oficiales y sus normatividades en los sujetos.

El carácter formativo, político e investigativo de las narraciones lo reconoce Jairo Gómez (2013) al comprender en su trabajo "los procesos de subjetivación política de jóvenes bogotanos configurados a través de sus experiencias y acontecimientos políticos, a partir de sus narrativas biográficas y políticas" (p. 18). Además, resalta el valor de la literatura como forma válida de conocer frente a las ciencias sociales y la filosofía que, dado su proceder canónico, no pueden adentrase en el sentido de la experiencia. Así, subraya la importancia de la imaginación y la ficción para provocar procesos de agenciamiento político. En el mismo sentido, Ruiz y Prada (2012) muestran la potencia 
de la narración para brindar herramientas para el ejercicio del poder y para producir un acercamiento con la alteridad.

En todo caso, puede señalarse que las metodologías biográfico-narrativas en educación se han constituido como prácticas formativas y de investigación que giran alrededor de la formación inicial de profesores o del quehacer docente. Para Sandra Rodríguez (2014), por ejemplo, la utilidad de las narrativas para los docentes no radica únicamente en que potencia sus prácticas, sino en "tomarlas como herramientas de reflexión político-pedagógica para ayudarnos a repensar la educación en América Latina" (p. 254). Así, con los relatos resignifican su trabajo educativo, la manera en la que ven a los estudiantes, a la escuela y "reconstruyen su identidad personal y la identidad del colectivo profesional y laboral al cual pertenecen" ( $p$. 256). De igual forma, la autora señala que, al igual que ocurre con la subjetividad, el lenguaje de la narración discurre sobre las prácticas al tiempo que las produce. De este modo, la metodología narrativa se caracteriza por ser útil para investigar e intervenir las realidades, dado que "contar una historia ayuda a procesar cuestiones que no estaban claras o no eran conscientes" (p. 265). Es en este proceso que los y las docentes "convierten su consciencia práctica en discursiva a través de la narración, la ponen en tensión, la componen y recomponen, la objetivan, la fijan en escritura, la comunican, la critican" (p. 267).

En el mismo camino, para Verónica Weber (2005) los relatos son herramientas de formación subjetiva que ayudan a los educadores a actuar de manera reflexiva al tomar como base su pensamiento práctico. Para la autora, es de vital importancia, para alcanzar este objetivo, que se den procesos de reescritura y socialización de las narraciones. En este escenario, se pone sobre la mesa el valor de los relatos para mirarse a sí mismo y a los demás, pues "buscamos con los relatos que los docentes transformen su conocimiento respecto de su práctica profesional y que en el proceso de esa transformación se transformen a sí mismos" (p. 16). De este modo, los relatos dejan ver la interpretación que dan los sujetos de sí mismos, de los demás y de los contextos. De igual manera, la vuelta sobre la propia experiencia es crucial para entender y guiar la toma de decisiones.

Entonces, es claro el papel de las narrativas (auto)biográficas en espacios escolares como dispositivos de formación-investigación de subjetividades. Así, dado que dichas metodologías se fundamentan principalmente en una mirada hermenéutica dirigen la atención especialmente a la experiencia como eje de la producción de sentidos y significados. En todo caso, se presentan algunas limitaciones o problemáticas del uso de relatos (auto) biográficos en la escuela. Una de ellas es la señalada por Gil (1997) en cuanto que la escuela no puede convertir los temas 'íntimos, privados y familiares' en su objetivo principal, sino abordarlos como parte de proyectos pedagógicos amplios. También, es importante reconocer los sesgos ideológicos de los trabajos que buscan "liberar" a los sujetos. Sumado a esto, debe tenerse en cuenta, en la construcción de narrativas en marcos institucionales, que los sujetos narradores pueden caer en contar lo que el investigador-docente quiere "escuchar". Finalmente, es relevante señalar, frente a la formación de subjetividades por medio de narraciones, que "no hay necesariamente ninguna línea de causalidad entre conocer la entraña misma de la autobiografía del alumno y alcanzar a despertarle la conciencia e identidad sobre sí mismo" (Gil, 1997, p. 130). Sin embargo, el autor citado reconoce como potencialidad del uso de ficciones biográficas la posibilidad que dan a los estudiantes para que se proyecten en personajes que no tuvieron lugar, pero los configuran identitariamente. 
Entonces, este reconocimiento del potencial de los relatos para la formación está vinculado con el rescate de la voz de los propios sujetos. Pues, debereconocerseque, porejemplo, lasdecisiones curriculares y didácticas de los docentes están sustentadas tanto en experiencias personales como en las condiciones institucionales. Por ello, es primordial "conocer cuáles son las condiciones en que desarrollan su trabajo, con que normatividades funcionan y cómo afectan su trabajo, cómo son los "espacios" sociales, afectivos, morales, etc." (Flores et al., 2005, p. $3)$. De esta manera, con la reivindicación de las experiencias personales se hace frente a la instrumentalización de la escuela y con ello puede configurarse procesos de resistencia frente a las políticas que buscan su eliminación. "El registro, la sistematización escrita, el acopio y la difusión pública de experiencias, prácticas y saberes escolares contadas a través de la voz y palabra de los docentes constituyen, al mismo tiempo, una propuesta político-pedagógica para la escuela" (Ministerio de Educación Nacional y OEA, 2005, p. 22). En todo caso, no deja de observarse en la escuela que se mira con desdén a la vivencia escolar para posicionar a los saberes formalizados. Esto a pesar de que:

lo que los adultos en formación guardan en la escuela, lo que designan del periodo escolar como significativo para su trayecto de formación son, mucho más que los contenidos de conocimiento, las experiencias relacionales, afectivas, sociales de las cuales la escuela fue -para ellos-el campo, y cuya polaridad emocional marcó su relación con el aprendizaje y el saber. (Delory-Momberger, 2009, p. 28)

En suma, en la literatura revisada, puede observarse la posibilidad de abordar la narrativa como fenómeno, metodología de investigación y formativa. Aunque, su uso en espacios educativos apunta sobre todo a la formación inicial de docentes y en general a la esfera de los educadores. Lo que no implica que cada vez se extienda más al trabajo con estudiantes y otras poblaciones. En particular, las investigaciones dirigen la mirada al campo de la enseñanza aprendizaje a través del registro de prácticas y en menor medida se enfocan en la formación explícita de la subjetividad. Esto, con la salvedad de que el método al buscar el rescate de la experiencia primaria opera como formador de subjetividad. De igual modo, los investigadores e investigadoras resaltan el carácter ético-político de las metodologías (auto) biográfico-narrativas de acuerdo con su carácter crítico que implica la producción conjunta del conocimiento. Por último, puede resaltarse que en los reportes de investigación-formación son escasas las reflexiones sobre la implementación misma de las herramientas de construcción de información. Así, se da prioridad a mostrar referentes conceptuales 0 categorías de análisis, describir instrumentos de recolección de investigación y hallazgos, pero no el cómo de la investigación. Por ejemplo, las instrucciones dadas a los participantes para construir los relatos y las problemáticas que hayan surgido en ese momento.

\section{LA VOLUNTAD DE FICCIÓN Y LA NARRACIÓN COMO ELEMENTOS DE FORMACIÓN DE LAS SUBJETIVIDADES POLÍTICAS: UNA APROXIMACIÓN A LOS REFERENTES CONCEPTUALES}

La investigación implementada tuvo como eje de reflexión la formación de subjetividades políticas a partir de herramientas (auto)biográficonarrativas en la escuela. Así, dado que las nociones centrales de subjetividad y narración son de difícil delimitación se requirió un punto nodal que las conectara. De este modo, la ficción hizo las veces de configuradora de la trama desarrollada. De ahí que, el trabajo se enfocó en explorar el papel de la ficcionalidad -como elemento narrativo- en la formación política. 
Este objetivo se desarrolló en el plano teórico y práctico.

La propuesta tuvo como suelo ontológico y teórico-político algunos de los planteamientos de las filosofías de Nietzsche y Ricoeur. Con base en estos, se pensó la subjetividad política desde la narrativa y la ficción. En este sentido, se encuentra el vínculo de la ficción, en los dos autores, para plantear la posibilidad de que mediante la fantasía o la imaginación creadora el sujeto pueda transfigurarse.

Pues bien, para pensar la subjetividad política desde la filosofía de Nietzsche es necesario comprenderla como una respuesta a la racionalidad metafísica moderna y por tanto a la voluntad de nada. Así, Conill (1997) define dicha filosofía como una hermenéutica genealógica que se encarga de realizar una crítica del modo de valorar metafísico. Pues, este modo de valoración se sustenta en la negación de la vida, en el nihilismo: "la creencia en la absoluta falta de valor, es decir, en la falta de sentido" (Nietzsche, 2008, p. 221). Para hacerle frente a este amor a la nada, que se encarna también en el cristianismo, Nietzsche le apuesta a establecer valores y sentidos desde la óptica de la vida, del cuerpo y de la tierra.

Este proyecto denominado transvaloración de todos los valores permite ver algunos de los elementos centrales de la filosofía de Nietzsche en su último periodo: la genealogía, la hermenéutica, la fisiología y la política. Precisamente, Conill (1997) resalta el carácter político del pensamiento nietzscheano al postular que la transvaloración es una 'empresa política' "porque tiene como resultado la transformación de las estructuras de poder y las formas de existencia" (p. 174). De este modo, la lucha por el dominio de la tierra es leída como una 'guerra hermenéutica', esto es, una disputa entre interpretaciones por imponer el sentido dominante. En este contexto de ideas, la preocupación de Nietzsche "se centra en la administración de la tierra y en la educación del hombre" (Conill, 1197, p. 189).

Las anteriores nociones están inscritas en la comprensión de la voluntad de poder como un campo de confrontación entre fuerzas por imponer una valoración de la vida. De hecho, para Nietzsche, la vida es definida como voluntad de poder. Esto quiere decir que, no es posible comprender la existencia al margen de las relaciones de poder: una fuerza no puede ser, sino en relación con otras fuerzas. Esta comprensión de la voluntad de poder como un escenario de confrontación entre diferentes perspectivas implica la existencia de un agonismo interpretativo. Esto involucra una defensa radical de la diferencia, de la singularidad y de la otredad. Ahora bien, la continua producción de interpretaciones de la voluntad de poder hace que sea comprendida como voluntad de ficción. Así, la existencia debe entenderse como un proceso infinito de producción de máscaras y el hombre como constituido por una 'conducta estética'.

Con base en los elementos expuestos es posible configurar una subjetividad política consciente del carácter ficcional y relacional de la existencia (Parra, 2019). Así, pensar la formación de la subjetividad política desde Nietzsche, sobre todo desde la voluntad de ficción (Parra, 2021), implica remitirse a la creación de nuevos sentidos desde la óptica de la vida y a la posibilidad de trasfiguración de la propia subjetividad. Esta, debe reconocerse, se encuentra anclada en el cuerpo, los instintos y las pasiones como elementos centrales de su constitución. Además, el marco relacional conflictivo permite comprender la política y la formación política por fuera de los registros tradicionales, tanto como abre paso a una racionalidad no lógica, sino narrativa. Tomar como base la ficcionalidad del cuerpo para reflexionar sobre lo político rompe 
los límites tradicionales de construir la verdad y con ello se da la posibilidad de configurar nuevas categorías para pensar la realidad.

Ahora bien, el sustrato ontológico relacional de la propuesta permitió postular, desde el campo narrativo, que no hay relatos absolutamente individuales (así se hagan en primera persona). Cuando nos narramos el otro está presente como el suelo concreto de nuestras experiencias. Empero, a pesar de que no podemos concebir la vida sin la alteridad, Walter Benjamín (1998) y Jorge Larrosa (2003) denuncian que se ha diluido la forma comunicativa que se sustenta en las narraciones configuradas desde las vivencias compartidas. Esto ocurre, pues hemos perdido la capacidad para tener experiencias, para comunicarnos y relacionarnos con los otros. De este modo, es necesario hacerse la pregunta por cómo lograr que se dé nuevamente el encuentro con la otredad a partir de las narraciones. Precisamente, la ficción como elemento narrativo permite que los sujetos reinterpreten su pasado y se transfiguren por medio de existencias imaginadas. En concreto, por medio de la construcción conjunta y la circulación de relatos -en los que se ficcionalizan las vivencias- es posible configurar experiencias y por tanto subjetividades.

Entonces, se afirma que los relatos y narraciones son dispositivos de formación de subjetividadespolíticas. Justamente, Ricoeur señala el fuerte vínculo que existe entre la configuración del relato a través de la trama y la configuración de la misma subjetividad. Es en ese sentido que, el filósofo francés define la subjetividad como identidad narrativa. Pues, Ricoeur comprende al hombre como un ser 'enmarañado en historias' no contadas que, precisamente, al realizar la selección de los acontecimientos para configurar una historia se da forma a sí mismo. De este modo, "la historia da cuenta del hombre" (Ricoeur, 2006, p. 19).
Este paralelo entre la construcción de la trama y la configuración subjetiva lleva a Ricoeur a pensar la vida humana desde la concordancia discordante del relato y la discordancia concordante del tiempo. Es decir, entre el hecho de que el relato intente hacer concordar lo discordante de los acontecimientos temporales en la trama y la aparente discordancia del continuo fluir del tiempo. Así, por medio de la inteligencia narrativa damos forma a nuestra identidad en una unidad con sentido pese al devenir constante de los acontecimientos. En consecuencia, la "subjetividad no es ni una serie incoherente de acontecimientos ni una sustancia inmutable inaccesible al devenir. Esa es el tipo de identidad que solamente la composición narrativa puede crear gracias a su dinamismo" (Ricoeur, 2006, p. 21).

De igual forma, Ricoeur establece un paralelo entre la dinámica de sedimentación e innovación de la obra literaria y el dinamismo de la identidad narrativa. Esto, implica que podemos leernos desde el lente de la cultura a la que pertenecemos. A su vez, por medio de la ficción podemos resistir a la fuerza de la identidad absoluta que intenta petrificarnos en el tiempo. Así, por medio de la ficción podemos desviarnos del mundo normatizado. "la relación más paradójica del arte con la realidad sería incomprensible si el arte no des-compusiera y no re-compusiera nuestra relación con lo real" (Ricoeur, 2002, p. 21). Esta transformación de la realidad por medio del relato puede efectuarse mediante la lectura, pues existe una acción transfiguradora del texto sobre lector en el momento de recepción de la obra.

\section{ME EN-VIDEO CON LAS NORMAS ESCOLARES ME EN-VIDEO CON LA ESCUELA}

Tomando como base el hecho ontológico de que el ser humano se forma relacional y conflictivamente, tanto como que al narrarse se configura su identidad, construí la estrategia 
didáctica Me en-video con las normas escolares, me en-video con la escuela. Así, en ella, tuve en cuenta el sustrato epistemológico que nos dan las metodologías (auto)biográfico narrativas, esto es, el hecho de que los relatos se consideren como instrumentos válidos de construcción y recolección de conocimiento. Dicha estrategia tuvo como propósito que los y las estudiantes trasfiguraran sus experiencias escolares y a sí mismos (as) por medio del uso de herramientas narrativas. De la misma manera, que cambiaran las miradas sobre sí mismos y los otros, tanto como la manera en la que se relacionan. Con la estrategia busqué construir reflexiones sobre su interpretación del mundo, en el marco de las relaciones de poder, para que la transformaran de manera creativa y crítica.

El término "en-videarse", que es usado coloquialmente por los y las estudiantes, lo empleo en este caso como sinónimo de "meterse" en el video. Esto implicó que reflexionaran sobre sus formas de ser y estar en la escuela para que las representaran por medio de relatos escritos y audiovisuales. Así, los ejercicios narrativos se configuraron en relación con sus experiencias escolares en torno a las normas de la escuela. "En-videar-se" con y en la escuela significa volver a mirar de manera reflexiva las formas en las que están y son con los otros en la cotidianidad.

En este sentido, la estrategia didáctica tuvo como objetivo formar una consciencia que les permitiera reflexionar sobre la manera en que se relaciona con los otros en el escenario de las relaciones de poder. O sea, dirigir la mirada críticamente a las formas activas o pasivas que tienen al pensar y actuar frente a sí mismos y a los demás. Este enfoque reflexivo que parte de lo relacional en el marco del poder y las jerarquías sociales es lo que entiendo como un modo político de pensar o una 'consciencia de poder'. Con esta se busca que los y las participantes en el trabajo pudieran transfigurar las prácticas de ser y estar en el contexto escolar. De este modo, las narrativas buscaban ser la herramienta para que pensaran su pasado, su presente y proyectaran otros mundos en los que se imaginaran nuevas formas de habitar con los otros: la descripción y transfiguración de su identidad.

Ahora bien, para construir las bases teóricas y prácticas de la estrategia didáctica tuve en cuenta los postulados epistemológicos y ontológicos expuestos. Con base en estos, elaboré cinco principios pedagógicos acordes con las metodologías (auto)biográfico narrativas en educación. El primero de ellos señala que mediante las narrativas es posible desnaturalizar, a partir de la reflexión crítica, los fenómenos abordados. Así, Mannay (2017) señala que "la producción de datos visuales y narrativos brinda la oportunidad de combatir la familiaridad, además de las jerarquías y las relaciones de poder inherentes, presentes en los lugares de investigación" (p. 71). Por ello, se buscó que los estudiantes reflexionaran y desnaturalizaran las maneras en las que se relacionaban con las normas escolares.

En segundo lugar, las narrativas generan un acercamiento a la vida personal (temporal) que produce experiencias a la vez que la posibilidad de que el sujeto se transfigure. Este principio se sustenta en la defensa que hace Nietzsche de la vida y la cotidianidad como dignos de atención filosófica. Así, la vida debe abordarse desde una mirada temporal no metafísica que permita inscribir en el torrente del devenir las confrontaciones por el sentido como parte de las dinámicas de las relaciones de poder. Este reconocimiento del carácter temporal del relato está relacionado con el modo de pensar narrativo, cercano a la consciencia histórica, como elemento constitutivo de la subjetividad política.

El tercero de los postulados reconoce que la producción conjunta y la circulación de los relatos dan paso al encuentro con los otros, aunque en el marco de la conflictividad 
inherente a las relaciones humanas. De esta forma, se afectan también las dinámicas de configuración de identidades tanto individuales como colectivas. Es así como, es posible que se transformen los modos de relacionarse antagónicos en agonistas. Es decir que, el otro deje de verse como un enemigo a eliminar y sea aceptado como un adversario legitimo sin el cual no podemos ser. Así, se potencia la defensa de la singularidad y la diferencia.

El cuarto de los principios indica que el trabajo con las herramientas narrativas influye en la expresividad de los y las estudiantes. Así, el uso puntual de estos instrumentos puede llevar a que se desarrollen nuevas maneras de narrarse y por tanto de comprenderse. En este punto, la ficcionalidad del relato cumple un papel fundamental al servir de base para que los sujetos reinterpreten su pasado, resignifiquen su presente y proyecten su futuro. Esto, pues la ficción del texto produce la expansión de los horizontes (Ricoeur, 2002).

Finalmente, presento la idea de que los relatos (auto)biográficos fungen como herramientas de politización. Pues, las narraciones, entre otras cosas, producen sentidos y pueden transfigurar las estructuras de poder. A través de los relatos los sujetos se ven interpelados a tomar una posición, tanto como a hacerse cargo de su propio destino. En este sentido, el carácter ético político de las narraciones implica la necesidad de que el estado de cosas no se mantenga estático.

Ahora bien, en términos prácticos, la estrategia didáctica se implementó en tres movimientos. En el primero de ellos, mi vida en la escuela, acciones en relación con las normas escolares, se trabajó en la elaboración de una "carpeta de vida" que contenía los relatos sobre las experiencias con las normas escolares de los y las estudiantes. Estos fueron complementados con dibujos, entrevistas a compañeros y familiares. En el segundo movimiento, mi vida narrada en movimiento: mi experiencia con las normas escolares, se elaboraron filminutos sobre sus experiencias. Finalmente, en el tercer movimiento, ficcionalizando la escuela, proyecciones del poder, se configuraron videos grupales en los que se imaginaron la escuela del futuro y su manejo de las normas en la escuela. En estos se tuvo en cuenta las historias personales. Cada movimiento implicó unos objetivos pedagógicos e investigativos que se desarrollaron paralelamente.

\section{NARRACIONES (AUTO)BIOGRÁFICAS EN LA ESCUELA. CONFIGURACIÓN Y CIRCULACIÓN DE RELATOS COMO ENCUENTRO CONSIGO MISMO Y CON LA ALTERIDAD.}

Para presentar los resultados de investigación realicé una interpretación, en términos políticos o de relaciones de poder, de las construcciones narrativas o respuestas dadas por los y las estudiantes en relación con las normas escolares. El tipo de análisis utilizado se denomina horizontal o comparativo (Bolívar, et al., 2001). Pues, a partir de los ejes temáticos previamente elaborados y de los que emergen en el trabajo de campo se buscan convergencias y divergencias que hacen las veces de unidades de sentido en las narraciones. Así, la descripción de lo que pasó en el aula con las herramientas biográficas la realizo desde los pensamientos y palabras del grupo de participantes en la investigación ${ }^{2}$. De esta manera, mediante el 'arte

\footnotetext{
$2 \quad$ Este modo de interpretación que está cercano a los analítico parece alejarse de una presentación de resultados acorde con lo narrativo y que fragmenta los relatos; sin embargo, el análisis narrativo no implica la nulidad de la sistematización rigurosa de los datos. En todo caso, señalo que como parte de la presentación de los resultados se elaboraron dos historias de vida que dan cuenta de la transformación operada por los relatos en dos de las estudiantes y que les permitió cambiar su mirada y forma de ser con los otros. En una de las historias una estudiante relata cómo cambió su mirada sobre la compañera al escuchar sus experiencias de vida y en la otra historia se puede ver cómo una de las participantes realiza una catarsis al poder contar a los compañeros algunos de los eventos que marcaron su vida. Precisamente, la estudiante de la primera historia cambia su mirada distante y poco amigable de la protagonista de la segunda historia.
} 
del bricolaje' configuro un relato e interpreto los resultados: "interpretar es dar significado a los hechos o datos, en un proceso de reconstruir, trasladar o encajar distintas piezas, en un buen bricolaje" (Bolívar, et al., 2001, p. 206).

Para poder explorar los límites y potencialidades de las narrativas (auto)biográficas se reflexionaba específicamente sobre lo que pasó en el momento de narrarse, tanto como de compartir o no los relatos. Así, se realizó el énfasis en la incidencia que tuvieron las prácticas narrativas en las perspectivas sobre sí mismos, sobre los otros y la manera en la que transformaron sus acciones cotidianas. En general, puede señalarse que los estudiantes se referían a sus experiencias en términos de bueno o malo, correcto o incorrecto. En este sentido, aunque se les señaló que el proyecto no buscaba "mejorar" los comportamientos en la escuela, tendían a mostrar como en el caso de encontrar una acción reprochable la ajustarían a los considerado correcto dentro de las normas escolares oficiales. Esto, de acuerdo con el escenario de aplicación del trabajo y a que el docente investigador trabaja en el mismo como coordinador de convivencia se consideró como parde del hecho de que los estudiantes se narran desde lugares "políticamente correctos". También, puede responder a que se narren desde lo que el mundo adulto espera escuchar.

En todo caso, puede señalarse que el grupo de estudiantes tendió a hablar más sobre sí mismos en general que sobre su relación con las normas. Esto, pues, a veces, se les presentaba como un tema abstracto. En todo caso, se evidencia que lograron darse cuenta de cosas que, aunque están en el diario vivir son naturalizadas. En este camino, por ejemplo, fue determinante la mirada del otro para que descubrieran cosas de sí mismos. Este darse cuenta lo reconstruí en tres niveles que se observan en las narraciones: en el primero se muestra que mediante los relatos pudieron tematizar elementos normativos y determinar su función en el entramado institucional; en el segundo, se vislumbra una reflexión frente a sus acciones que se acompaña de las valoraciones bueno o malo, correcto o incorrecto; finalmente, se encuentra la afirmación de un pensamiento que podría denominarse crítico, esto es, la reflexión sobre el sentido por el que se actúa o no.

\section{DIRIGIR LA MIRADA A LAS NORMAS ESCOLARES. LAS NORMAS PARA NORMALIZAR Y COHESIONAR A LA SOCIEDAD}

Como resultado primario de dirigir la mirada a las acciones bajo la lupa de las normas escolares se encuentra la posibilidad de pensar sobre lo que se hace en la vida diaria. Sin embargo, la reflexión encontraba un límite en la aprehensión de las normas como dispositivo social y no solamente como un elemento al que se remite como parte de la lógica entre mandar y obedecer impuesta por los adultos. En este contexto, se desenvuelven en un juego entre el premio y el castigo en el que se encuentran pasivamente sin ninguna reflexión profunda sobre el por qué obedecer.

Ahora bien, para permitir un mejor acercamiento de los y las estudiantes con el tema de las normas escolares se recurrió a la ejemplificación constante. Así, se intentó diferenciar las normas explícitas u oficiales de las implícitas; sin embargo, la mayoría de los relatos hacían referencia a las normas instituidas oficialmente. En consecuencia, se ven como recurrente valorar una acción en términos morales, así como la necesidad de corregirse y acercarse al parámetro de comportamiento: "que caí en cuenta que estaba haciendo mal y lo corregi" (Estudiante, Cuestionario) ${ }^{3}$.

$3 \quad$ Al finalizar cada uno de los movimientos se aplicaba un cuestionario al curso con el fin de preguntar sobre las impresiones e impacto del uso de herramientas narrativas y configuración de los relatos. Estas mismas preguntas se discutían más ampliamente en el grupo focal. 
En todo caso, es importante señalar que el ejercicio de tematizar las normas escolares no fue significativo para la totalidad de estudiantes. Algunos (as) mencionaban que fue un ejercicio importante para aprender más sobre las normas o reconocer su importancia en las dinámicas sociales. En otro nivel, se reconoció el papel de las normas para cohesionar la sociedad y mecanismo de regulación de conflictos. De igual forma, se reconoció que algunas de las normas estaban para proteger, en el caso del colegio, a los mismos estudiantes. En otro nivel, no solo reconocían el papel de las normas, sino que explicitaban la necesidad de cambiar, aunque la mayoría de las veces para adecuarse a lo considerado como aceptable.

Así, pude observar que los y las estudiantes se comprenden como parte de un sistema normativo ya establecido y sobre el que tienen poca injerencia. En concordancia, la imagen que tienen de sí mismos se reduce a considerarse como cumplidor o infractor de las normas. Aunque, también reconocen que los y las docentes están regidas por normatividades que les son impuestas y señalan que parte de su labor consiste en hacerlas cumplir. Es decir, identifican "una cadena de normatividades (...) una jerarquía de relaciones" (docente investigador). A partir de estas ideas, se trabajó la posibilidad de que se identificaran como productores de normas o como seres capaces de tomar una posición frente a las mismas. En este sentido, se intentó identificar las normas implícitas y que regulan, de alguna manera, las relaciones primarias entre estudiantes. Frente a la posibilidad de comprenderse como productores de normas señalaban que no percibían en el colegio los espacios para participar en la construcción de la organización escolar. Es decir, no ven un poder horizontal y la posibilidad de incidir en las decisiones que se toman.
En todo caso, se evidenció en las reflexiones que las normas sociales, ya sean explícitas o implícitas, producen formas de normalización de las subjetividades. Esto es, se reprueban o aprueban formas de ser y de estar que moldean a los individuos. En este sentido, lo correcto o lo incorrecto actúan como formas de parametrizar o "como estereotipos de lo que está bien en una persona o está mal" (estudiante, grupo focal 3). Esto pude observarlo en una de las intervenciones con el grupo focal en que una de las estudiantes señaló que sufría de acoso escolar por no responder a los parámetros "normales": "no tenía una apariencia o no he tratado de buscarla como otras niñas que, por ejemplo, yo tuve ese apodo de patito feo, de la enana, la delgada, cosas así y eso me bajó mucho la autoestima" (estudiante, grupo focal 3). Frente a esto, la estudiante señalaba los sentimientos de soledad por la exclusión producida, pero encontraba en compañeros que veían más allá de lo físico espacio para la confianza propia. En todo caso, indicaba que en el grupo de estudiantes, pues ingreso un año antes, no encontró un espacio para la integración y al contario sintió el rechazo.

\section{HABLA EL CAMELLO: OBEDECER LAS REGLAS}

"Hay muchas cosas pesadas para el espíritu, para el espíritu fuerte, paciente, en él habita la veneración: su fortaleza demanda cosas pesadas, e incluso las más pesadas de todas" (Nietzsche, 1992. p. 49).

En el análisis realizado se fijó la mirada en la manera en la que el acercamiento a las normas escolares produjo alguna transformación. Así, se identificaron posturas poco criticas frente al cumplimiento de las normatividades: "si porque nos hace pensar en no desobedecer las reglas" (Estudiante, cuestionario). En este sentido, se encontraron actitudes que apuntaban a la importancia de cumplir la norma por la norma. De este modo, el respeto por las reglas escolares 
es una premisa que guía sus comportamientos y por tanto cualquier acción que se salga de estas debe ser corregida. Con base en esto, aparecen el orden y el cuidado como elementos que configuran al sujeto aplicado. Esta situación estaba vinculada al sentimiento por el deber cumplido: "significa para mí mucho o con la relación con las normas me gusta respetar las normas" (Estudiante, cuestionario).

De este modo, la reflexión con base en la experienciapropiaymediadaporlasherramientas narrativas sobre las normas escolares permitió, en principio, que los estudiantes se situaran como cumplidores o infractores de estas. Así, se logró que reflexionaran sobre su subjetividad en relación con la manera en la que actuaban en el colegio, aunque de manera acrítica. En todo caso, pensar en las repercusiones del actuar es la base para que se dé una transformación en el ámbito práctico. Así, algunas de las reflexiones incluían la presencia del otro como sujeto digno de respeto, pues se denota que el incumplimiento de algunas normas implica una afectación del otro. En este punto puede señalarse que el término respeto aparecía mayormente cuando los estudiantes estaban en grado octavo y responsabilidad era más usado en grado once. Ahora bien, un elemento que comenzaba a vislumbrarse como parte de una subjetividad más crítica fue la pregunta por el sentido de la manera en la que se actuaba. Sin embargo, a pesar de que el objetivo de la intervención no era formar sujetos cumplidores de la norma por la norma se presentaron inevitablemente posturas de sometimiento a las reglas escolares.

\section{APARECE EL LEÓN: ROMPER LAS CADENAS.}

"Pero en lo más solitario del desierto tiene lugar la segunda transformación: el león se transforma aquí en espíritu, quiere conquistar su libertad como se conquista una presa, y ser señor de su propio desierto" (Nietzsche, 1992, p. 50).
Este tipo de abordajes sobre las normas, aunque no fue el predominante, estuvo antecedido por el hecho de que en el espacio de los talleres se pudiera criticar de manera abierta a las obligaciones escolares. Esta posibilidad que se denomina crítico-expresiva surge al permitir que los y las estudiantes dijeran de manera abierta aquello que sienten y piensan frente a la organización escolar. Esto es, enunciar la verdad de sus pensamientos sin miedo a represalias. Esto también estuvo vinculado con la oportunidad de que propusieran nuevas formas de habitar la escuela.

Sin embargo, se observa una tendencia de las reflexiones hacia el cumplimiento de las normas. Esto, a pesar de que se puso sobre la mesa que la idea del ejercicio no era "juzgar" sus comportamientos, que no existía la posibilidad de sanción por lo relatado y estuviera fuera del marco normativo. De hecho, en los talleres de aprestamiento se planteó la posibilidad de defender un comportamiento que no fuera considerado como adecuado. Así mismo, se discutió que frente a las normas consideradas como injustas podría tomarse una posición de no acatamiento. Ahora bien, algunos integrantes del curso afirmaron la necesidad de romper las reglas, pero indicaba que lo hacian por aburrimiento o experimentar. De igual modo, señalaron que estaban en desacuerdo con los límites en el uso del espacio escolar, pero lo justificaron al mismo tiempo dada la imprudencia de algunos estudiantes.

\section{PROYECCIONES FICCIONALES: ¿HABLA EL NIÑO?}

"Inocencia es el niño, y olvido, un nuevo comienzo, un juego, una rueda que se mueve por sí misma, un primer movimiento, un santo decir sí" (Nietzsche, 1992, p. 51).

El potencial de las herramientas narrativas, mediante la configuración y circulación de los relatos, para transformar a los sujetos se 
evidenció en el acercamiento que produjo con los otros y la mirada reflexiva sobre sí mismos. Aunque, los mismos participantes señalaron el límite de los ejercicios al argumentar que no bastaba con palabras para cambiar, sino eran llevadas las intenciones a la acción. De este modo, también se vieron las posturas que mostraban que no hubo ningún cambio en ellos al realizar las prácticas narrativas. Sin embargo, más allá de la efectividad de los instrumentos narrativos para trasmutar las subjetividades, se evidenció en los discursos la resistencia al cambio, por ejemplo, por considerar que no era necesario.

De este modo, formativamente es relevante que comiencen a pensarse como sujetos en transformación constante. Para ello, la producción de relatos permite que, mediante la identificación de los momentos de ruptura, se entiendan como individuos históricamente constituidos. Esto, lo pueden entender, como cambios atribuidos a la edad, pero que responden a decisiones y determinaciones propias. En la reflexión sobre los ejercicios realizados algunos (as) reconocieron que había transformaciones en su actuar en la escuela. Dichos cambios no solo correspondían como su manera de resolver los problemas, sino también con la forma de ver a los otros. Así, los ejercicios narrativos permitieron identificar estas transformaciones al menos en el campo discursivo.

Ahora bien, en el momento final de la estrategia, en el que por grupos de estudiantes debían elaborar un video colectivo presentando algún problema relacionado con las normas escolares en la escuela del futuro, se evidenció que no lograron crear situaciones por fuera de los marcos normativos tradicionales. Esto no quiere decir que sus trabajos no tuvieran elementos futuristas, pero si que no hubo ninguna forma novedosa de afrontar los problemas de convivencia. De hecho, apuntaban a la necesidad de docentes más estrictos, mejores controles que implicaran hacer cumplir las normas, esto es, mayor "disciplina". De igual manera, señalaban la importancia de estudiantes más concentrados en sus estudios y del diálogo para solucionar sus problemas. En uno de los guiones de los videos que se enfocaba en la problemática de la inasistencia escolar era manejada de manera tradicional: llamado de atención, exclusión del estudiante del salón de clase, remisión a coordinación o citación de acudiente. En este sentido, era la institución quien debía tomar cartas en el asunto por medio de sus mecanismos de regulación.

\section{CONCLUSIONES}

A partir del abismo entre el pensamiento, el discurso, la acción y la imagen de esta es posible señalar que no basta con la introducción de las herramientas narrativas en el aula para la formación de la subjetividad política. Así, es necesario que estén articuladas a unos objetivos pedagógicos, formativos y políticos. De igual manera, es necesario que exista un colectivo que se movilice socialmente para fortalecer los elementos constitutivos de la subjetividad política. Sin embargo, los instrumentos narrativos (auto)biográficos y biográficos en el aula permiten, desde la ficción, que los sujetos interpelen su pasado, vitalicen su presente y proyecten su futuro como parte de un proceso de transfiguración identitaria.

En general, la producción individual y colectiva de los relatos, tanto como su circulación, permitió que los y las estudiantes volvieran sobre sí, salieran de sí y se encontraran con la alteridad de sus compañeros (as). Sin embargo, para que esto ocurra deben existir las condiciones de enunciación institucionales y las subjetivas. Es decir, se requieren espacios para que las palabras y los gestos circulen, al tiempo que la disposición de los sujetos a contar y escuchar las historias. De este modo, si se eliminan las barreras en la comunicación, se da la posibilidad para que pase algo que transforme a los 
espacios educativos y a los sujetos. Así, este acercamiento a los otros a partir de sus historias hace parte de lo que podemos denominar una razón o reflexión narrativa con tinte político.

Estos límites pueden estar dados por la extrañeza que produce hablar de la vida cotidiana en la escuela. Lo que se debe, entre otros factores, a la mirada "academicista" de algunos docentes que dan prioridad a los contenidos de las disciplinas frente a la experiencia. Esta perspectiva también puede atravesar a los estudiantes atrapados en una mirada instrumental del aprendizaje. Por otro lado, el manejo que tengan de la oralidad, la escritura y las herramientas audiovisuales cuenta para la configuración de los productos. En consecuencia, se hacen necesarios ejercicios de reescritura reflexiva como parte de los procesos de construcción de los relatos. Por tanto, construir las condiciones para que las narraciones circulen se constituye en una acción política que lleva a transformar los tiemposespacios institucionales, junto con las formas de ser de la comunidad escolar.

\section{REFERENCIAS BIBLIOGRÁFICAS}

Bolívar, A. Domingo, J y Fernández, M. (2001). La investigación biográfico-narrativa en educación. Enfoque y metodología. Editorial la Muralla: Madrid.

Benjamín, W. (1998). Para Una crítica de la violencia y otros ensayos. Iluminaciones IV. Madrid: Taurus.

Conill, J. (1997). El poder de la mentira. Nietzsche y la política de la transvaloración. Tecnos. Madrid.

Delory-Momberger. (2015). La condición biográfica. Ensayos sobre el relato de sí en la modernidad avanzada. Medellín: Editorial Universidad de Antioquia.
Flores, R. et al., (2015). La cultura Profesional de los Docentes en enseñanza Secundaria: Un Estudio Biográfico. Archivos Analíticos de Políticas Educativas. Vol. 13, Núm. 49. Diciembre, págs. 1-26. Recuperado en:

ht tps: / / w w w.red a I y c.org / pdf/2750/275020513049.pdf

Gil, F. (1997). Educación y narrativa: la práctica de la autobiografía en la educación. Teoría Educativa. 9. Ediciones Universidad de Salamanca, págs. 115136. Recuperado en:

https://revistas.usal.es/index.php/1130-3743/ article/view/3134/3162

Gómez, Esteban. (2013). Testigos de sí mismos. Narrativas políticas de jóvenes bogotanos. Universidad Distrital: Bogotá.

Huchim, A y Reyes Ch. (2013). La investigación biográfico narrativa, una alternativa para el estudio de los docentes. En: Revista electrónica Actualidades Investigativas en Educación. Vol. 13, núm. 13. Marzodiciembre, págs. 1-27. Recuperado en:

https://www.redalyc.org/pdf/447/44729878019. pdf

Larrosa, Jorge. (2003). La experiencia de la lectura. Estudios sobre literatura $y$ formación. México: Fondo de cultura económica.

Mannay, D. (2017). Métodos visuales, narrativos y creativos en investigación cualitativa. Madrid. Narcea. 
Ministerio de Educación ciencia y tecnología y Organización de los Estados Americanos Agencia Internacional para la Cooperación y el Desarrollo. (2005). La documentación narrativa de experiencias pedagógicas. Una estrategia para la formación de docentes. Argentina.

Murillo, G. (2016). La investigación biográficonarrativa en educación en Colombia siglo XXI. Universidad de Antioquia, Medellín. Tesis doctoral.

Nietzsche, F. (2008). Fragmentos póstumos (1885-1889) Volumen IV. Tecnos. Madrid. (1992). Así habló Zaratustra. Alianza Editorial: Madrid

Parra, E. (2021). Pensar la formación de la subjetividad política a partir de la voluntad de ficción de Friedrich Nietzsche. Estudios Políticos (Universidad de Antioquia), 62, págs. Recuperado en:

https://revistas.udea.edu.co/index.php/ estudiospoliticos/article/view/344334

(2020). Voluntad de ficción, subjetividades políticas y narrativas (auto) biográficas. Una experiencia de formación política con estudiantes de secundaria. Bogotá: Universidad de los Andes. Tesis doctoral. Recuperado en:

https://repositorio.uniandes.edu.co/bitstream/ handle/1992/48385/u833949. pdf?sequence $=1$

(2019). Representaciones y narraciones formativas de la alteridad. La subjetividad política como escenario de relación y de conflicto. Análisis, 51 (95), págs. 389-416. Recuperado en: https://revistas.usantotomas.edu.co/index.php/ analisis/article/view/4756/pdf

Rodríguez, S. (2014). Las narrativas como estrategia de formación docente. En revista interamericana, Vol. 7 , núm. 2. Julio-diciembre, págs. 251-270. Recuperado en:

https://revistas.usantotomas.edu.co/index.php/ riiep/article/view/1957/2067

Ricoeur. P. (2006). La vida: un relato en busca de narrador. Ágora (papeles de filosofía) 25/2, págs. 9-22. Recuperado en: https://minerva.usc.es/xmlui/ bitstream/handle/10347/1316/Ricoeur. pdf?sequence $=$

(2002), Del texto a la acción. Ensayos de hermenéutica II. México. Fondo de Cultura Económica.

Ruiz, A. y Prada, M. (2012). La formación de la subjetividad política. Propuestas y recursos para el aula. Paidós: Buenos Aires.

Velasco, J. (2019). El método biográfico y las historias de vida. Su utilidad en la investigación en enfermería. Index de Enfermería, (edición Digital); 27. Recuperado en: http://www.index-f. com/index-enfermeria/27revista/27 articulo_25-30.php.

Weber, Verónica. (2005). El relato de experiencias como estrategia para la formación docente. (Ponencia), presentada en: $\mathrm{V}$ congreso Internacional Virtual de Educación, Argentina. Recuperado en:

http://sedici.unlp.edu.ar/bitstream/ handle/10915/24628/Documento completo.pdf? sequence $=1$ \&isAllowed $=y$ 